\title{
Analysis of Anthropometric Measurements and Dietary Intake in Patients Undergoing a Multi-Professional Osteoarthritis Education Program (PARQVE-Project Arthritis Recovering Quality of Life by Means of Education)
}

\author{
Nádia Lucila Rocha Brito, Marcia Uchoa de Rezende*, Thiago Pasqualin, \\ Gustavo Constantino de Campos, Renato Frucchi, Marcelo Issao Hissadomi, \\ Alexandre Felicio Pailo, Olavo Pires de Camargo \\ Department of Orthopedicsand Traumatology, Hospital das Clínicas, Faculdade de Medicina da \\ Universidade de São Paulo, São Paulo, Brazil \\ Email: nadianutri@uol.com.br, ”murezende@uol.com.br, thiagopasqualin@gmail.com, \\ gustavoccampos@terra.com.br, renatofrucchi@hotmail.com,ma_issao@hotmail.com, \\ alexandrepailo@yahoo.com.br, olapcama@uol.com.br
}

Received 10 December 2015; accepted 22 February 2016; published 26 February 2016

Copyright (C) 2016 by authors and Scientific Research Publishing Inc.

This work is licensed under the Creative Commons Attribution International License (CC BY).

http://creativecommons.org/licenses/by/4.0/

(c) (i) Open Access

\begin{abstract}
Background: Osteoarthritis $(\mathrm{OA})$ has a major impact on mobility and the loss of productivity of patients, especially knee $\mathrm{OA}(\mathrm{KOA})$. Obesity is one of the main risk factors for the incidence and prevalence of KOA. Weight loss alone decreases pain and improves quality of life and functional scores. Objective: To use BMI, body fat, and calorie intake to measure the effect of a multi-professional educational program on patients with KOA and correlate these measurements with subjective questionnaire results. Methods: A total of 198 patients undergoing standard treatment for KOA were randomized to 4 groups. All groups received written and video information regarding KOA. Three groups (1, 2 and 3$)$ also attended two days of lectures 1,2 and 3 months apart, respectively, whereas group 4 did not. Each group was divided into subgroups A (bimonthly telephone calls) and B (no telephone calls). All patients were evaluated at baseline and at one year for BMI, waist-hip ratio (WHR), percentage of body fat, and calorie intake and with the WOMAC, LEQUESNE, VAS and SF-36 questionnaires. Results: The WHR showed $89.4 \%$ of android obesity at baseline and
\end{abstract}

${ }^{*}$ Corresponding author.

How to cite this paper: Brito, N.L.R., et al. (2016) Analysis of Anthropometric Measurements and Dietary Intake in Patients Undergoing a Multi-Professional Osteoarthritis Education Program (PARQVE-Project Arthritis Recovering Quality of Life by Means of Education). Open Journal of Orthopedics, 6, 32-45. http://dx.doi.org/10.4236/ojo.2016.62006 
$87.9 \%$ at one year without improvement $(p=0.38)$. Body fat decreased by $0.44 \%$ regardless of group or subgroup. Calorie intake was different between groups $(p=0.019)$ according to phone calls and follow-up $(p=0.03)$. BMI and body fat percentage were correlated with the WOMAC, WOMAC pain, VAS and LEQUESNE scores at baseline and at reassessment. Weight was correlated with the baseline results of the WOMAC pain $(r=0.175, p=0.014)$, VAS $(r=0.155, p=0.029)$, LEQUESNE $(r=0.161, p=0.023)$, SF-36-PCS $(r=-0.186, p=0.009)$ and SF-36-MCS $(r=0.155, p=$ $0.029)$ scores and with the one-year results of the WOMAC $(r=0.155, p=0.029)$ and WOMAC pain $(r=0.151, p=0.034)$ scores. Conclusion: The multi-professional treatment program had very little impacts on the percentage of body fat. This improvement was independent of classes, telephone calls, or improvements in pain, function and quality of life. Calorie intake improvement was influenced by telephone calls and classes but was not associated with objective measurements of/or changes in weight, BMI, or body fat percentage or with subjective improvements.

\section{Keywords}

\section{Diet Therapy, Osteoarthritis, Body Mass Index, Knee, Education}

\section{Introduction}

Osteoarthritis (OA) is the most frequent form of arthritis and the main cause of chronic disability [1]. The disease incidence and prevalence increases with low socio-economic level, longevity and the presence and duration of obesity [2]-[7]. Knee OA (KOA) is among the most prevalent and disabling types of OA [8].

In Brazil, according to information from the National Household Sample Survey (PNAD) 2008, the prevalence of arthritis or rheumatism corresponds to $5.7 \%$ of Brazil's population [9]. Longevity and obesity are increasing in Brazil [10] [11], and 50.2\% of Brazilians have no education or incomplete primary education [12]. As a result, this population has a great potential for OA and KOA, with their consequences of decreased quality of life [13].

Currently, OA is irreversible [14]. Optimal management of OA requires a combination of non-pharmacological and pharmacological modalities [15]. Patients must decrease their weight and increase their physical activity and strength [15]-[18].

Accumulation of metabolic syndrome (MetS) components appears to be associated with a higher intensity of knee pain independent of weight; therefore, appropriate treatment of MetS may be helpful for subjects with KOA [19]. Self-management, education and provision of information about OA and its treatment are widely promulgated as core recommendations for the treatment of OA hip and knee in recent guidelines [15] [17] [18]. This information can be given by a booklet alone, a self-management course, by a physician as part of a consultation, in small groups or delivered by multidisciplinary team [20]-[24]. A previous positive experience with a multi-professional team educational program in osteoporosis [24] led us to idealize a similar (multi-professional team) program on OA.

The aim of this study is to evaluate the effects of multi-professional education in patients with knee osteoarthritis (KOA) by anthropometric measurements, including variations of the percentage of body fat, calorie intake, and waist-hip ratio, and by pain, functional and quality of life questionnaires and to correlate the obtained results.

\section{Methods}

\subsection{Design}

This prospective, randomized controlled trial followed the guidelines of the CONSORT statements for randomized controlled trials and non-drug treatments [25].

\subsection{Participants}

Care providers included one nutritionist, seven orthopedic surgeons, four psychologists, three social workers, 
five occupational therapists, three physical therapists and two physical educators, all of whom were volunteers or staff at the Orthopedic Institute, Hospital das Clínicas, University of São Paulo.

Patients had to meet the following criteria: outpatient of 45 years of age or older with KOA according to the American College of Rheumatology clinical and radiological definition [26]; no rheumatoid arthritis or any other rheumatologic disease other than OA; undergoing standard care for OA for the previous six months; knee pain rated above $30 \mathrm{~mm}$ on a numerical scale and necessitating drug treatment; no neurological problems; and the ability to understand, agree with and sign the informed consent and the questionnaires, regardless of education level. The exclusion criteria included not being evaluated by the nutritionist, undergoing surgery during the study, missing classes, participating in another program with nutritional education, or enrolling in another clinical trial.

\subsection{Intervention}

Participants were undergoing standard care for the treatment of KOA at the Osteometabolic Diseases Group, Department of Orthopedics and Traumatology, Hospital das Clínicas, University of São Paulo. By November 2011, 306 patients were receiving standard care for KOA, which included being followed by orthopedic surgeons and submitting to blood tests for metabolic syndrome (with a referral to a general practitioner for clinical control) and calcium metabolism, X-rays, densitometry and more specific images (ultrasound and magnetic resonance image (MRI)) depending on symptoms. All patients were prescribed diacerhein. Paracetamol and codeine were offered for pain. Muscle relaxants and magnesium were prescribed if patients complained of cramps. Non-steroidal anti-inflammatory drugs (NSAIDs) were used for short periods of time for severe pain. Vitamin D3 and calcium supplements were prescribed according to blood levels and bone densitometry results. When present, osteoporosis was treated with alendronate. Based on X-rays, orthotics, such as custom-made hand orthotics and valgus or varus insoles, canes, and walkers were prescribed. Patients were referred to physical therapy and acupuncture when they suffered impaired mobility and pain. Of these 306 patients, 228 met the inclusion criteria and were interested in participating in the study.

At enrollment, patients were evaluated for anthropometric data, BMI and waist-hip ratio; had seven skin folds measured to obtain the percentage of body fat; and were asked for details on their diet over the previous last 24 hours to estimate calorie intake. Patients were also asked to respond to the VAS (Visual Analogue Scale), WOMAC $^{\mathrm{TM}}$, Lequesne and SF-36 questionnaires [27]-[29]. All patients had plain radiographs of their knees, including weight-bearing anterior-posterior, lateral and patellar axial views. Three orthopedic surgeons examined all radiographs to classify the severity of OA according to Kellgren and Lawrence [30]. Disagreements between the first two surgeons were resolved by the third.

Participants were randomly allocated in four groups (1 to 4, according to days of lectures) and 2 subgroups (A and B, according to telephone calls) of 28 or 29 participants each. Three groups had two days of lectures on OA. All groups received printed material to read and a video with all the lectures. Groups 1, 2, and 3 attended lectures one, two and three months apart, respectively. Group 4 received the educational material only. Subgroup A received bimonthly telephone calls, and subgroup B did not receive telephone calls. Patients in each group were asked to come to the hospital on two specific Saturdays according to the intervals of their group.

The program, explained partially in the short-term results [31], comprised two days (from 7:00 to 17:00 hours) of lectures, workshops and meals. The nutritionist's program on the first intervention day was explanatory and emphasized the importance of a small, colorful, frequent (every 3 hours), fiber-rich, low-fat and low glycemic index diet, as well as the importance of drinking at least 2 liters of liquid per day. During the second intervention, the nutritionist reviewed the diet with the patients following the slide sequence shown in the first class and answered questions on each specific topic.

Both days of lectures had 30- to 60-minute intervals at 9:30 (30 minutes), 13:00 (1 hour) and 16:00 (30 minutes) for meals with the diet suggested by the nutritionist. Patients followed the recommendations of eating every 3 hours and of consuming colorful foods, 3 to 5 fruits a day (exceptions when restricted by clinicians), whole wheat grains, and 8 glasses of water/tea or juice (one fruit/glass only) a day with the exception of patients with fluid restrictions.

The physicians called patients in subgroup A two months after the lecture and then every other month until the 1-year reassessment. Patients were asked about pain, medication, diet, occupational therapy, and social and/or physical activity and frequency. They were reminded to read the booklet or watch the DVD as well as to exercise at least three times a week (preferably daily) and to follow correct diet, social and occupational habits. 
Twelve months after the final lecture or after receiving the educational material, patients returned for an evaluation. Again, patients were evaluated for anthropometric data; seven skin folds; previous 24-hour diet for calorie intake estimation; and LEQUESNE, WOMAC ${ }^{\mathrm{TM}}$, VAS, and SF-36 questionnaires. The evaluators were blinded to the allocation.

\subsection{Simple size}

This is a pilot study to evaluate the best (time-wise) intervention to add multi-professional education to KOA clinical treatment. The authors aimed to have 30 patients in each group.

\subsection{Randomization}

Randomization was performed by a computer-generated program (available at http://www.randmization.com/). Patients were randomly allocated in one of the 4 groups.

\subsection{Blinding}

There was no difference in demographics between groups. Groups 1, and 3 had classroom instructions from professionals as well as audio-visual and written instructions, which group 4 also received. Patients knew when signing the informed consent that groups would differ according to time between classes or lack of classes and telephone calls or lack thereof. Evaluators did not know the group to which each patient belonged. Two secretaries scheduled appointments, classes, and material retrieval and plotted the questionnaire results in Excel.

\subsection{Statistical Analysis}

Anthropometric and dietary measures have been described by groups, telephone calls and evaluation moments using summary measures (mean, standard deviation and 95\% confidence interval). The values between groups, the presence or lack of telephone calls, and moments of evaluation were compared using analysis of variance with repeated measures with three factors, followed by Bonferroni multiple comparisons to compare the groups, telephone calls and evaluation moments as needed.

Pearson correlations were made between functional scales and anthropometric measurements and calorie intake at baseline and at the one-year reassessment.

The existence of a marginal association between the type of obesity at baseline and at reassessment was verified using the McNemar test.

Changes in pain, function and quality of life scores were compared according to the type of obesity at baseline and at reassessment using Student's t-test (Kirkwood and Sterne, 2006).

The tests were conducted at a $5 \%$ significance level.

\section{Results}

Of the 306 patients assessed for eligibility, 246 met the inclusion criteria. However, only 228 agreed to participate (Figure 1). Groups 2A, 2B, 3A and 3B had 28 patients each; 29 patients were assigned to each of the remaining groups. Sixteen patients missed classes and did not complete the study (because of lost interest, weather conditions that prevented access to the hospital or scheduling conflicts). At this point, subgroups varied from 25 $(1 \mathrm{~A}, 2 \mathrm{~B})$ to $29(1 \mathrm{~B}, 4 \mathrm{~B})$ participants. At the one-year reassessment, four patients had died (one each from groups $1 \mathrm{~A}, 1 \mathrm{~B}, 3 \mathrm{~B}$ and $4 \mathrm{~A})$. One patient had undergone total knee replacement (group $1 \mathrm{~A})$. Six patients $(2 \mathrm{~A}$ and $3 \mathrm{~A}=$ one patient, $4 \mathrm{~A}$ and $4 \mathrm{~B}=$ two patients each) missed the evaluation and, when called, decided not to continue in the study (either because they were no longer interested or because they were too far away to attend follow-ups). One patient from group 1A and two from group 4B missed the reassessment (Figure 1). Thirty patients were ultimately excluded from the study, with 8 from group 1, 10 from group 4, and 12 from groups 2 and 3.In summary, the study ended with 198 patients. Groups 1 to 3 ended with 50 patients each (1A: 22; 1B: 28; 2A: 25; 2B: 25; 3A: 25; 3B: 25) and group 4 with 48 patients (4A: 23 and 4B: 25).

Table 1 shows the WHR, weight, BMI, percentage of body fat and calorie intake according to group, telephone calls and moments of evaluation. ANOVA results for the comparison of anthropometric measurements and calorie intake between groups, telephone calls and evaluation moments showed that the waist/hip ratio average differed between groups over time $(p=0.027$, Table 2$)$. However, this difference could not be specifically 


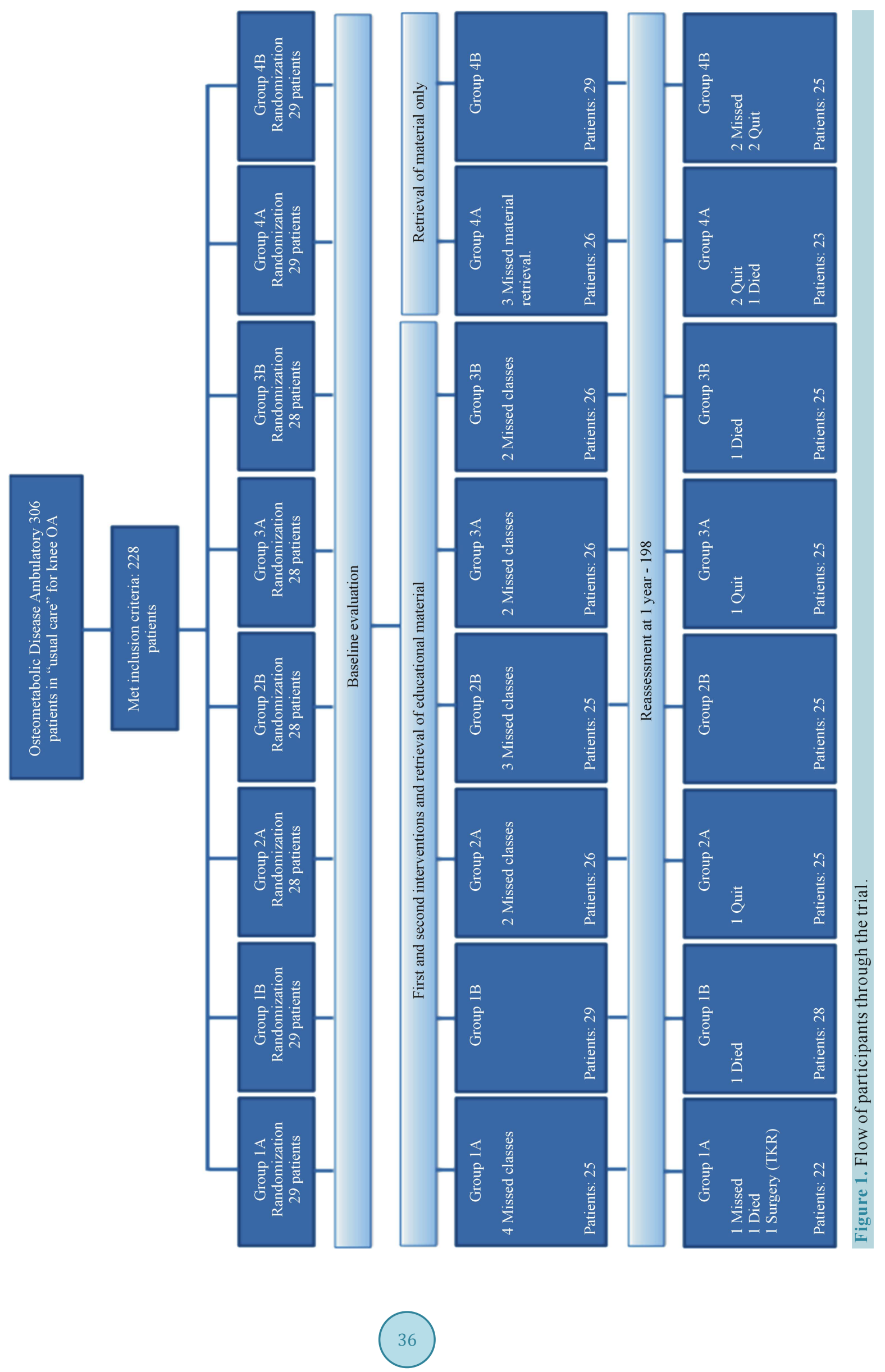




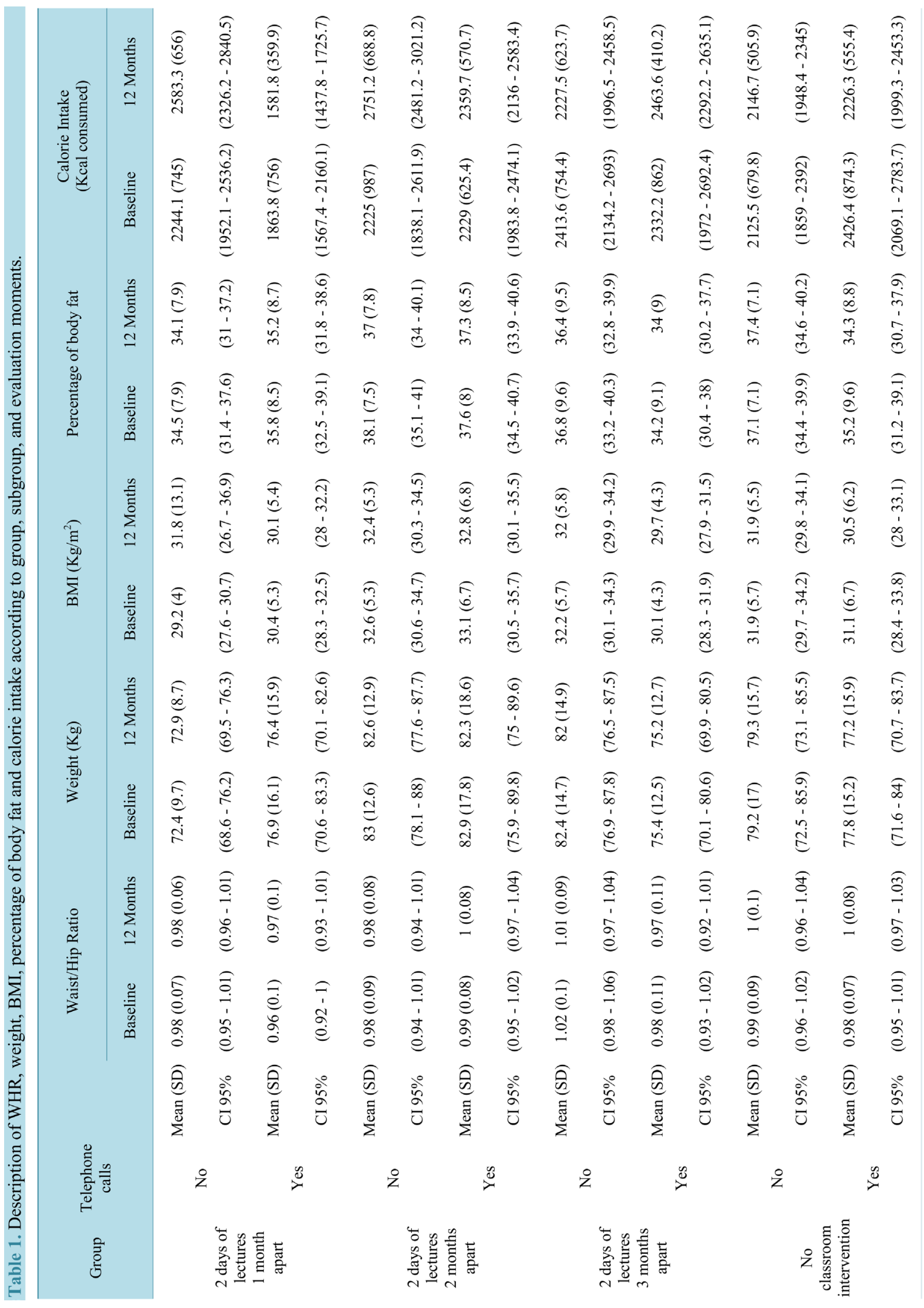


Table 2. ANOVA results for comparisons of anthropometric measures and calorie intake between groups, telephone call and evaluation moments.

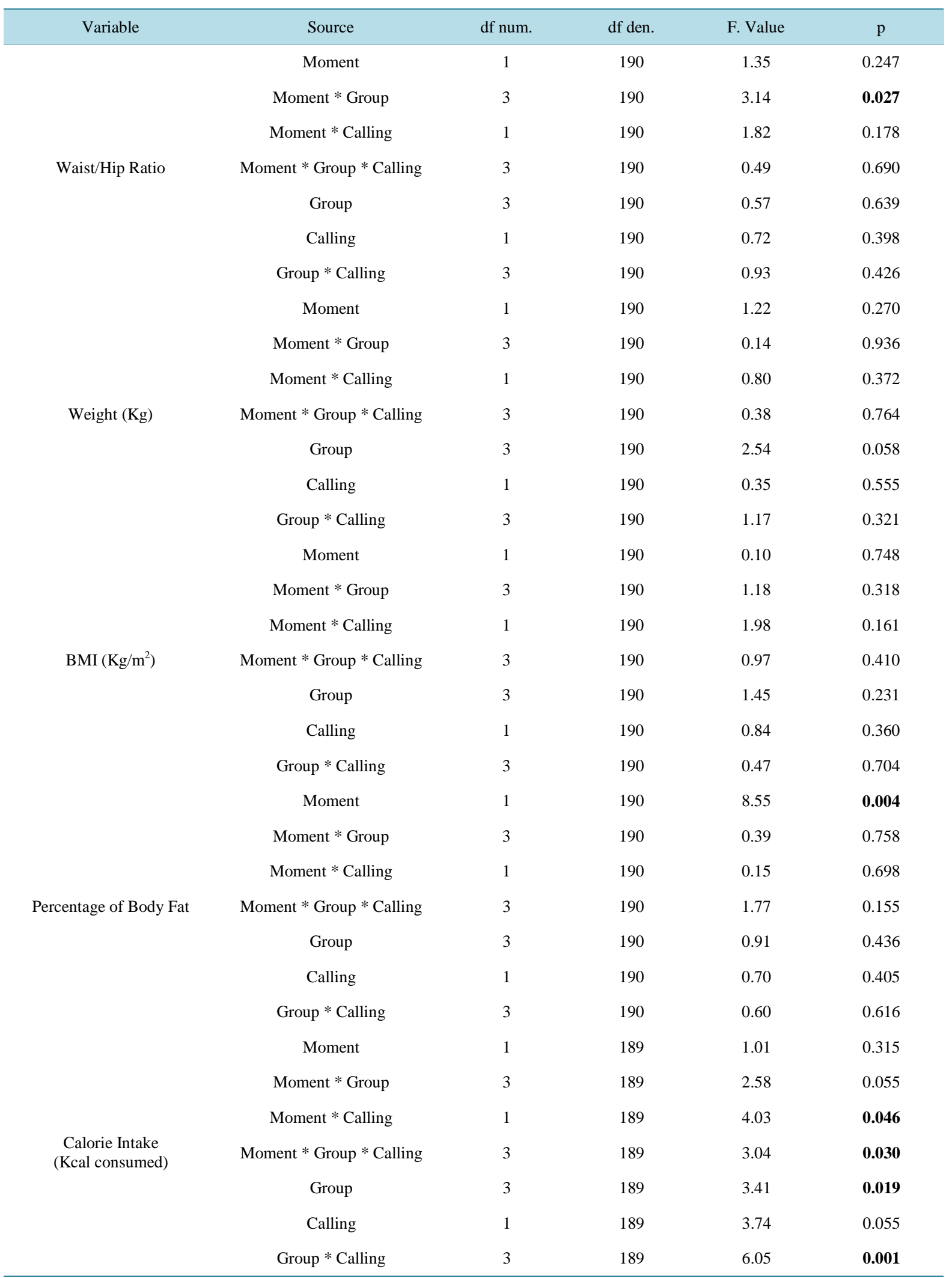


identified between any two groups (Table 3). Of the 198 patients, 72 (36.36\%) decreased their BMI (45 by one point and 27 by more than two points); 72 had no BMI change; and 54 (27.27\%) increased their BMI by at least one point, despite guidance to decrease weight.

The average body fat percentage differed between moments of evaluation regardless of group or subgroup ( $\mathrm{p}$ $=0.004$, Table 2$)$. The average body fat diminished by $0.44 \%(\mathrm{CI} 0.424,0.456, \mathrm{p}<0.001)$ from baseline to the one-year reassessment.

Caloric intake was influenced by group, subgroup, and moment of evaluation (Table 2). The group with classes 1 month apart and with telephone calls had a lower average calorie intake at 12 months $(\mathrm{p}<0.001$, Table 4) than the same group without telephone calls, and at 12 months, this group exhibited a significant lower average calorie intake than groups with class intervals of 2 and 3 months $(p=0.008$ and $p=0.001$, respectively, Table 4).

Type of obesity did not change significantly from baseline to the one-year reassessment, as shown in Table 5 . Type of obesity (android or gynecoid) had no difference in respect to pain, function and quality of life scores at baseline and at the one-year reassessment $(p>0.05$, Table 6$)$.

Table 7 shows that, weight, BMI and body fat percentage correlated with pain, function and quality of life scores at baseline and at reassessment. Although there were significant correlations between anthropometric measurements and pain, function and quality of life scores, none of the correlations was greater than 0.25 , indicating a low correlation between these measurements.

Change in caloric intake was inversely correlated with the change in WOMAC pain $(r=-0.141, p=0.048)$. Again, the correlation value was very close to zero, although it was statistically significant (Table 8).

Table 3. Result of the multiple comparison between groups and moments for WHR.

\begin{tabular}{|c|c|c|c|c|c|c|c|}
\hline \multirow{2}{*}{ Group/Moment } & \multirow{2}{*}{ Comparisons } & \multirow{2}{*}{$\begin{array}{c}\text { Mean } \\
\text { Difference }\end{array}$} & \multirow{2}{*}{ S.E. } & \multirow{2}{*}{ df } & \multirow{2}{*}{$\mathrm{p}$} & \multicolumn{2}{|c|}{$95 \% \mathrm{CI}$} \\
\hline & & & & & & Lower & Upper \\
\hline 2 days of lectures 1 month apart & Baseline - 12 months & -0.003 & 0.004 & 1 & $>0.999$ & -0.017 & 0.010 \\
\hline 2 days of lectures 2 months apart & Baseline - 12 months & -0.008 & 0.004 & 1 & $>0.999$ & -0.021 & 0.005 \\
\hline 2 days of lectures 3 months apart & Baseline - 12 months & 0.011 & 0.004 & 1 & 0.339 & -0.003 & 0.024 \\
\hline No classroom intervention & Baseline - 12 months & -0.012 & 0.004 & 1 & 0.163 & -0.025 & 0.002 \\
\hline \multirow{7}{*}{ Baseline } & Lectures 1 month apart - Lectures 2 months apart & -0.011 & 0.018 & 1 & $>0.999$ & -0.066 & 0.045 \\
\hline & Lectures 1 month apart - Lectures 3 months apart & -0.027 & 0.018 & 1 & $>0.999$ & -0.082 & 0.029 \\
\hline & Lectures 1 month apart - no classroom intervention & -0.015 & 0.018 & 1 & $>0.999$ & -0.071 & 0.041 \\
\hline & & & & & & & \\
\hline & Lectures 2 months apart - Lectures 3 months apart & -0.016 & 0.018 & 1 & $>0.999$ & -0.072 & 0.040 \\
\hline & Lectures 2 months apart - no classroom intervention & -0.005 & 0.018 & 1 & $>0.999$ & -0.061 & 0.051 \\
\hline & Lectures 3 months apart - no classroom intervention & 0.011 & 0.018 & 1 & $>0.999$ & -0.045 & 0.068 \\
\hline \multirow{6}{*}{12 Months } & Lectures 1 month apart - Lectures 2 months apart & -0.015 & 0.018 & 1 & $>0.999$ & -0.071 & 0.040 \\
\hline & Lectures 1 month apart - Lectures 3 months apart & -0.013 & 0.018 & 1 & $>0.999$ & -0.068 & 0.043 \\
\hline & Lectures 1 month apart - no classroom intervention & -0.024 & 0.018 & 1 & $>0.999$ & -0.080 & 0.032 \\
\hline & Lectures 2 months apart - Lectures 3 months apart & 0.003 & 0.018 & 1 & $>0.999$ & -0.053 & 0.058 \\
\hline & Lectures 2 months apart - no classroom intervention & -0.008 & 0.018 & 1 & $>0.999$ & -0.064 & 0.048 \\
\hline & Lectures 3 months apart - no classroom intervention & -0.011 & 0.018 & 1 & $>0.999$ & -0.067 & 0.045 \\
\hline
\end{tabular}


Table 4. Results of the multiple comparisons of calorie intake between groups, telephone call and evaluation moments.

\begin{tabular}{|c|c|c|c|c|c|c|c|}
\hline \multirow{2}{*}{ Group/Moment/Calling } & \multirow{2}{*}{ Comparisons } & \multirow{2}{*}{$\begin{array}{c}\text { Mean } \\
\text { Difference }\end{array}$} & \multirow{2}{*}{ S.E. } & \multirow{2}{*}{ df } & \multirow{2}{*}{$\mathrm{p}$} & \multicolumn{2}{|c|}{$95 \%$ IC } \\
\hline & & & & & & Lower & Upper \\
\hline Lectures 1 month apart - w/o telephone calls & Baseline - 12 months & -339.2 & 160.9 & 1 & $>0.999$ & -907.2 & 228.8 \\
\hline Lectures 1 month apart - with telephone calls & Baseline - 12 months & 285.9 & 163.1 & 1 & $>0.999$ & -289.8 & 861.6 \\
\hline Lectures 2 months apart - w/o telephone calls & Baseline - 12 months & -526.2 & 160.9 & 1 & 0.129 & -1094.1 & 41.8 \\
\hline Lectures 2 months apart - with telephone calls & Baseline - 12 months & -130.8 & 160.9 & 1 & $>0.999$ & -698.7 & 437.2 \\
\hline Lectures 3 months apart - w/o telephone calls & Baseline - 12 months & 186.1 & 152.1 & 1 & $>0.999$ & -350.6 & 722.8 \\
\hline Lectures 3 months apart - with telephone calls & Baseline - 12 months & -131.5 & 171.6 & 1 & $>0.999$ & -736.9 & 474.0 \\
\hline Educational material only - w/o telephone calls & Baseline - 12 months & -21.2 & 160.9 & 1 & $>0.999$ & -589.1 & 546.8 \\
\hline Educational material only - with telephone calls & Baseline - 12 months & 200.2 & 167.8 & 1 & $>0.999$ & -392.0 & 792.3 \\
\hline Lectures 1 month apart - Baseline & w/o telephone calls - with telephone calls & 380.4 & 193.9 & 1 & $>0.999$ & -304.1 & 1064.8 \\
\hline Lectures 1 month apart - 12 months & w/o telephone calls - with telephone calls & 1005.4 & 195.7 & 1 & $<0.001$ & 314.6 & 1696.3 \\
\hline Lectures 2 month apart - Baseline & w/o telephone calls - with telephone calls & -3.9 & 193.9 & 1 & $>0.999$ & -688.3 & 680.5 \\
\hline Lectures 2 month apart - 12 months & w/o telephone calls - with telephone calls & 391.5 & 193.9 & 1 & $>0.999$ & -292.9 & 1075.9 \\
\hline Lectures 3 month apart - Baseline & w/o telephone calls - with telephone calls & 81.4 & 195.3 & 1 & $>0.999$ & -608.0 & 770.8 \\
\hline Lectures 3 month apart - 12 months & w/o telephone calls - with telephone calls & -236.1 & 195.3 & 1 & $>0.999$ & -925.5 & 453.3 \\
\hline Educational material only - Baseline & w/o telephone calls - with telephone calls & -300.9 & 198.1 & 1 & $>0.999$ & -1000.1 & 398.2 \\
\hline Educational material only - 12 months & w/o telephone calls - with telephone calls & -79.6 & 198.1 & 1 & $>0.999$ & -778.7 & 619.6 \\
\hline \multirow{6}{*}{ Without telephone calls - Baseline } & Lectures 1 month apart - Lectures 2 months apart & 19.1 & 193.9 & 1 & $>0.999$ & -665.3 & 703.5 \\
\hline & Lectures 1 month apart - Lectures 3 months apart & -169.5 & 188.7 & 1 & $>0.999$ & -835.3 & 496.4 \\
\hline & Lectures 1 month apart - Educational material only & 118.6 & 193.9 & 1 & $>0.999$ & -565.8 & 803.0 \\
\hline & Lectures 2 months apart - Lectures 3 months apart & -188.6 & 188.7 & 1 & $>0.999$ & -854.4 & 477.3 \\
\hline & Lectures 2 months apart - Educational material only & 99.5 & 193.9 & 1 & $>0.999$ & -584.9 & 783.9 \\
\hline & Lectures 3 months apart - Educational material only & 288.1 & 188.7 & 1 & $>0.999$ & -377.8 & 953.9 \\
\hline
\end{tabular}

Table 5. Frequency of the type of baseline and one-year obesity and result in marginal association test.

\begin{tabular}{|c|c|c|c|c|c|}
\hline \multirow{2}{*}{$\begin{array}{l}\text { Type of } \\
\text { Obesity }\end{array}$} & \multicolumn{2}{|c|}{ Baseline } & \multicolumn{2}{|c|}{12 Months } & \multirow{2}{*}{$\mathrm{p}$} \\
\hline & $\mathrm{n}$ & $\%$ & $\mathrm{n}$ & $\%$ & \\
\hline Android & 177 & 89.4 & 174 & 87.9 & \multirow[b]{2}{*}{0.375} \\
\hline Gynecoid & 21 & 10.6 & 24 & 12.1 & \\
\hline
\end{tabular}

McNemar test. 
Table 6. Results of correlations between anthropometric measurements and calorie intake with pain, function and quality of life results at baseline and at one year.

\begin{tabular}{|c|c|c|c|c|c|c|c|c|c|c|c|c|c|c|c|}
\hline \multirow{2}{*}{ Variable } & \multirow{2}{*}{$\begin{array}{l}\text { Type of } \\
\text { obesity }\end{array}$} & \multicolumn{7}{|c|}{ Baseline } & \multicolumn{7}{|c|}{12 Months } \\
\hline & & Mean & SD & Median & Minimum & Maximum & $\mathrm{N}$ & $\mathrm{p}$ & Mean & SD & Median & Minimum & Maximum & $\mathrm{N}$ & $\mathrm{p}$ \\
\hline \multirow{2}{*}{ WOMAC } & Android & 1.57 & 17.80 & 2 & -35 & 60 & 177 & \multirow{2}{*}{0.963} & 1.62 & 17.67 & 2 & -34 & 60 & 174 & \multirow{2}{*}{0.879} \\
\hline & Gynecoid & 1.38 & 14.42 & 2 & -23 & 33 & 21 & & 1.04 & 16.05 & 2 & -35 & 33 & 24 & \\
\hline \multirow{2}{*}{$\begin{array}{l}\text { WOMAC } \\
\text { Pain }\end{array}$} & Android & 0.52 & 4.43 & 1 & -15 & 13 & 177 & \multirow{2}{*}{0.816} & 0.59 & 4.29 & 1 & -13 & 13 & 174 & \multirow{2}{*}{0.428} \\
\hline & Gynecoid & 0.29 & 3.65 & 0 & -5 & 9 & 21 & & -0.17 & 4.80 & 0 & -15 & 9 & 24 & \\
\hline \multirow{2}{*}{ VAS } & Android & 3.37 & 30.05 & 2 & -84 & 80 & 177 & \multirow{2}{*}{0.612} & 3.53 & 29.34 & 1.5 & -73 & 80 & 174 & \multirow{2}{*}{0.499} \\
\hline & Gynecoid & -0.10 & 24.98 & 0 & -41 & 59 & 21 & & -0.83 & 31.17 & 2 & -84 & 59 & 24 & \\
\hline \multirow{2}{*}{ Lequesne } & Android & -0.02 & 4.21 & 0 & -15 & 15 & 177 & \multirow{2}{*}{0.588} & -0.08 & 4.15 & 0 & -15 & 15 & 174 & \multirow{2}{*}{0.300} \\
\hline & Gynecoid & 0.50 & 3.07 & 0.5 & -4 & 8 & 21 & & 0.85 & 3.74 & 0.25 & -4 & 11 & 24 & \\
\hline \multirow{2}{*}{ SF-36 PCS } & Android & 0.86 & 9.09 & 0.8 & -27.6 & 28.5 & 177 & \multirow{2}{*}{0.563} & 1.07 & 8.87 & 0.95 & -27.6 & 28.5 & 174 & \multirow{2}{*}{0.156} \\
\hline & Gynecoid & -0.36 & 9.64 & -1.1 & -15 & 16.4 & 21 & & -1.75 & 10.73 & -1.2 & -23.7 & 16.4 & 24 & \\
\hline \multirow{2}{*}{ SF-36 MCS } & Android & 2.43 & 13.19 & 1.5 & -33 & 53 & 177 & \multirow{2}{*}{0.726} & 2.31 & 13.30 & 1.35 & -33 & 53 & 174 & \multirow{2}{*}{0.983} \\
\hline & Gynecoid & 1.38 & 10.45 & 1.8 & -15 & 28 & 21 & & 2.37 & 9.80 & 2.3 & -15 & 28 & 24 & \\
\hline
\end{tabular}

t-Student test.

Table 7. Results of correlations between anthropometric measurements and calorie intake with pain function and quality of life results at baseline and at the one-year reassessment.

\begin{tabular}{|c|c|c|c|c|c|c|c|c|c|c|c|c|c|}
\hline \multirow{2}{*}{ Correlation } & & \multicolumn{6}{|c|}{ Baseline } & \multicolumn{6}{|c|}{12 Months } \\
\hline & & WOMAC & $\begin{array}{l}\text { WOMAC } \\
\text { Pain }\end{array}$ & VAS & Lequesne & $\begin{array}{l}\text { SF-36 } \\
\text { PCS }\end{array}$ & $\begin{array}{l}\text { SF-36 } \\
\text { MCS }\end{array}$ & WOMAC & $\begin{array}{l}\text { WOMAC } \\
\text { Pain }\end{array}$ & VAS & Lequesne & $\begin{array}{l}\text { SF-36 } \\
\text { PCS }\end{array}$ & $\begin{array}{l}\text { SF-36 } \\
\text { MCS }\end{array}$ \\
\hline \multirow{3}{*}{$\begin{array}{l}\text { Waist/Hip } \\
\text { Ratio }\end{array}$} & $\mathrm{r}$ & 0.068 & -0.024 & 0.064 & 0.028 & 0.018 & 0.026 & 0.089 & 0.022 & 0.086 & 0.121 & -0.102 & 0.029 \\
\hline & $\mathrm{p}$ & 0.343 & 0.740 & 0.367 & 0.694 & 0.801 & 0.714 & 0.213 & 0.757 & 0.229 & 0.090 & 0.152 & 0.688 \\
\hline & $\mathrm{N}$ & 198 & 198 & 198 & 198 & 198 & 198 & 198 & 198 & 198 & 198 & 198 & 198 \\
\hline \multirow{3}{*}{ Weight } & $\mathrm{r}$ & 0.137 & 0.175 & 0.155 & 0.161 & -0.186 & 0.155 & 0.155 & 0.151 & 0.104 & 0.174 & -0.118 & 0.058 \\
\hline & $\mathrm{p}$ & 0.054 & 0.014 & 0.029 & 0.023 & 0.009 & 0.029 & 0.029 & 0.034 & 0.144 & 0.014 & 0.097 & 0.416 \\
\hline & $\mathrm{N}$ & 198 & 198 & 198 & 198 & 198 & 198 & 198 & 198 & 198 & 198 & 198 & 198 \\
\hline \multirow[t]{2}{*}{ BMI } & $\mathrm{p}$ & 0.021 & 0.005 & 0.031 & 0.027 & 0.004 & 0.633 & 0.014 & 0.005 & 0.035 & 0.011 & 0.124 & 0.553 \\
\hline & $\mathrm{N}$ & 198 & 198 & 198 & 198 & 198 & 198 & 198 & 198 & 198 & 198 & 198 & 198 \\
\hline \multirow{3}{*}{$\begin{array}{l}\text { Percentage } \\
\text { of body fat }\end{array}$} & $\mathrm{r}$ & 0.177 & 0.191 & 0.193 & 0.161 & -0.232 & -0.077 & 0.240 & 0.249 & 0.217 & 0.228 & -0.108 & -0.085 \\
\hline & $\mathrm{p}$ & 0.013 & 0.007 & 0.006 & 0.023 & 0.001 & 0.279 & 0.001 & $<0.001$ & 0.002 & 0.001 & 0.132 & 0.236 \\
\hline & $\mathrm{N}$ & 198 & 198 & 198 & 198 & 198 & 198 & 198 & 198 & 198 & 198 & 198 & 198 \\
\hline \multirow{3}{*}{$\begin{array}{l}\text { Calorie intake } \\
\text { (Kcal } \\
\text { consumed) }\end{array}$} & $\mathrm{r}$ & -0.096 & -0.127 & -0.136 & -0.050 & 0.020 & 0.074 & -0.034 & -0.070 & -0.088 & -0.068 & 0.037 & 0.005 \\
\hline & $\mathrm{p}$ & 0.177 & 0.073 & 0.056 & 0.485 & 0.783 & 0.299 & 0.634 & 0.328 & 0.218 & 0.340 & 0.608 & 0.943 \\
\hline & $\mathrm{N}$ & 198 & 198 & 198 & 198 & 198 & 198 & 197 & 197 & 197 & 197 & 197 & 197 \\
\hline
\end{tabular}


Table 8. Results of correlations between anthropometric measurements and calorie intake with changes in pain and functional scales.

\begin{tabular}{|c|c|c|c|c|c|c|c|}
\hline Correlations & & WOMAC & WOMAC Pain & VAS & Lequesne & SF-36 PCS & SF-36 MCS \\
\hline \multirow{3}{*}{$\begin{array}{c}\text { Waist/Hip Ratio } \\
\text { (baseline - } 12 \text { months) }\end{array}$} & $\mathrm{r}$ & 0.139 & 0.062 & 0.062 & 0.046 & 0.099 & 0.089 \\
\hline & $\mathrm{p}$ & 0.051 & 0.386 & 0.388 & 0.516 & 0.166 & 0.212 \\
\hline & $\mathrm{N}$ & 198 & 198 & 198 & 198 & 198 & 198 \\
\hline \multirow{3}{*}{$\begin{array}{c}\text { Weight } \\
\text { (baseline - } 12 \text { months) }\end{array}$} & $\mathrm{r}$ & 0.123 & 0.077 & 0.067 & 0.061 & 0.017 & 0.098 \\
\hline & $\mathrm{p}$ & 0.083 & 0.282 & 0.346 & 0.394 & 0.812 & 0.169 \\
\hline & $\mathrm{N}$ & 198 & 198 & 198 & 198 & 198 & 198 \\
\hline \multirow{3}{*}{$\begin{array}{c}\text { BMI } \\
\text { (baseline - } 12 \text { months) }\end{array}$} & $\mathrm{r}$ & -0.034 & 0.022 & 0.000 & -0.037 & 0.009 & 0.018 \\
\hline & $\mathrm{p}$ & 0.634 & 0.761 & 0.995 & 0.601 & 0.896 & 0.798 \\
\hline & $\mathrm{N}$ & 198 & 198 & 198 & 198 & 198 & 198 \\
\hline \multirow{3}{*}{$\begin{array}{l}\text { Percentage of body Fat } \\
\text { (baseline - } 12 \text { months) }\end{array}$} & $\mathrm{r}$ & 0.135 & 0.131 & 0.116 & 0.061 & 0.066 & 0.063 \\
\hline & $\mathrm{p}$ & 0.059 & 0.067 & 0.103 & 0.395 & 0.353 & 0.378 \\
\hline & $\mathrm{N}$ & 198 & 198 & 198 & 198 & 198 & 198 \\
\hline \multirow{3}{*}{$\begin{array}{c}\text { Calorie Intake } \\
\text { (baseline - } 12 \text { months) }\end{array}$} & $\mathrm{r}$ & -0.110 & -0.141 & -0.055 & 0.009 & -0.102 & -0.036 \\
\hline & $\mathrm{p}$ & 0.124 & 0.048 & 0.444 & 0.895 & 0.152 & 0.614 \\
\hline & $\mathrm{N}$ & 197 & 197 & 197 & 197 & 197 & 197 \\
\hline
\end{tabular}

\section{Discussion}

The purpose of this study was to objectively measure the effects of a multi-professional educational program for patients with knee OA by comparing weight, BMI, body fat percentage and calorie intake with subjective scores. The waist-hip ratio has been shown to correlate with KOA and WOMAC scores [32], but it did not in our study. This could be explained by the fact that our patients were in the obesity grade 1 range and the study that showed the android WHR in women to be related to KOA evaluated non-obese patients.

It has been said that weight loss alone decreases pain and improves quality of life and functional scores [16]. Patients in our study did reduce their weight, but not by the $6.1 \mathrm{~kg}$ described as necessary to improve pain [33]. However, our weight results did correlate (weakly) with the WOMAC, WOMAC pain and Lequesne results at baseline and at one year. Weight also correlated to baseline quality of life scores. Similarly, BMI improved little over a one-year period regardless of group or telephone calls, but it did correlate with the WOMAC, WOMAC pain, VAS and Lequesne results at baseline and at one year. Of the 198 patients who completed the study, 72 reduced their BMI by at least one point but only 27 decreased it by more than two points as a result of the program. All twenty-seven showed improvements in pain and/or function as reported by Bliddal [16].

The percentage of body fat improved in all patients regardless of group or subgroup. The body fat percentage improvement shows that patients gained lean body mass even if they did not lose weight, which may reflect increased physical activity, a reduced calorie intake and/or improved diet quality. The percentage of body fat also correlated with the WOMAC, WOMAC pain and Lequesne scores at baseline and at reassessment. Changes in calorie intake correlated inversely with changes in the WOMAC pain score, suggesting that the greater the reduction in calorie consumption, the less improvement in pain the patient had. Because this information is contrary to all published studies [15]-[18], one must question the reliability of the answers given by patients and rely more on objective data.

Patients were initially unaware of the relationship between their diet habits, their body composition and their symptoms. Their diets had excessive carbohydrates and saturated fat and very little fiber, i.e., their diet quality and quantity were poor. Changes in calorie intake at the one-year reassessment were influenced by group and subgroup. The group with classes one month apart showed a lower caloric intake than the other groups at the 
one-year reassessment. Those in group 1 who received bimonthly telephone calls also had a lower caloric intake than those in group 1 without telephone calls. Because this lower caloric intake was not reflected in weight loss or in a lower BMI or percentage of body fat, one must question the accuracy of their answers. Patients might have responded based on what the investigators taught but did not actually apply what they learned.

Our study has limitations. First, it is a pilot study. Almost 200 patients were distributed among 8 groups. Second, our patients were of a low socio-economic level. This implies difficulties in actually acquiring goodquality food, because carbohydrates are less expensive and more easily acquired, as well as difficulties in comprehension, but at the same time indicates that this population is our target. We must be able to help them based on this reality. Third, we did not monitor lipid and sugar levels. Blood testing was performed to verify metabolism disorders, but it was not the aim of the study to correlate anthropometric data with sugar and lipid blood levels. This could be performed in future studies. Fourth, we did not control physical activity levels to correlate our results. These data exist and can be analyzed on future studies. Roughly $2 / 3$ did not change or even increased BMI after the educational program. The program must be improved to reach a significant number of patients with clinically relevant improvements.

\section{Conclusion}

The multi-professional treatment program had very little impacts on the percentage of body fat. This improvement was independent of classes, telephone calls, or improvements in pain, function and quality of life. Calorie intake improvement was influenced by telephone calls and classes but was not associated with objective measurements of weight, BMI, or body fat percentage or with subjective improvements.

\section{Ethical Approval}

This prospective, randomized controlled trial was conducted under the principles of the Helsinki Declaration and approved by the Ethics Committee for the Analysis of Research Projects (CAPPesq) under protocol number 0622/11. Clinical Trials registration number: NCT01572051.

\section{Acknowledgements}

This study could not been performed without the voluntary help of Heloísa Ungaro, Paulo Dallari, Miriam Damaris Di Maio, Alípio Jose Gusmão dos Santos, and Pérola Grinberg Plapler; the secretaries (especially Suellen Lima, Natalia Borges, Rosilane Zaranelli Castro Dutra and Mercedes Alves Coutinho); the occupational therapist team; the social workers; the physical therapists; the psychologists; the physical educators; and the security staff of Hospital das Clínicas, Department of Orthopedics, Faculdade de Medicina Universidade de São Paulo.

This study was funded by both TRB Pharma TM-Brazil and the Department of Orthopedics and Traumatology, Hospital das Clínicas, University of São Paulo, neither of which had any role in the project design and implementation, data collection, analysis and interpretation, or manuscript writing.

\section{References}

[1] Sharma, L. and Kapoor, D. (2007) Epidemiology of Osteoarthritis. In: Moskowitz, R.W., Altman, R.D., Hochberg, M.C., Buckwalter, J.A., Goldberg, V.M., Eds., Ostearthritis, 4th Edition, Lippincott Williams \& Wilkins, Philadelphia, 3-26.

[2] Jorgensen, K.T., Pedersen, B.V., Nielsen, N.M., Hansen, A.V., Jacobsen, S. and Frisch, M. (2011) Socio-Demographic Factors, Reproductive History and Risk of Osteoarthritis in a Cohort of 4.6 Million Danish Women and Men. Osteoarthritis and Cartilage, 19, 1176-1182. http://dx.doi.org/10.1016/j.joca.2011.07.009

[3] Dixon, T., Shaw, M., Ebrahim, S. and Dieppe, P. (2004) Trends in Hip and Knee Joint Replacement: Socioeconomic Inequalities and Projection of Need. Annals of the Rheumatic Diseases, 63, 825-830. http://dx.doi.org/10.1136/ard.2003.012724

[4] Mahomed, N.N., Barrett, J., Katz, J.N., Baron, J.A., Wright, J. and Losina, E. (2005) Epidemiology of Total Knee Replacement in the United States Medicare Population. The Journal of Bone \& Joint Surgery, 87, 1222-1228. http://dx.doi.org/10.2106/JBJS.D.02546

[5] Blagojevic, M., Jinks, C., Jeffery, A. and Jordan, K.P. (2010) Risk Factors for Onset of Osteoarthritis of the Knee in Older Adults: A Systematic Review and Meta-Analysis. Osteoarthritis and Cartilage, 18, 24-33. http://dx.doi.org/10.1016/j.joca.2009.08.010 
[6] Felson, D.T., Anderson, J.J., Naimark, A., Walker, A.M. and Meenan, R.F. (1988) Obesity and Knee Osteoarthritis. The Framingham Study. Annals of Internal Medicine, 109, 18-24. http://dx.doi.org/10.7326/0003-4819-109-1-18

[7] Wills, A.K., Black, S., Cooper, R., Coppack, R.J., Hardy, R., Martin, K.R., et al. (2012) Life Course Body Mass Index and Risk of Knee Osteoarthritis at the Age of 53 Years: Evidence from the 1946 British Birth Cohort Study. Annals of the Rheumatic Diseases, 71, 655-660. http://dx.doi.org/10.1136/ard.2011.154021

[8] Parmet, S., Lynm, C. and Glass, R.M. (2003) JAMA Patient Page. Osteoarthritis of the Knee. JAMA, 289, 1068. http://dx.doi.org/10.1001/jama.289.8.1068

[9] Brasil Instituto Brasileiro de Geografia e Estatística (2008) Pesquisa nacional por amostra de domicílios. IBGE, Rio de Janeiro. http://www.ibge.gov.br/home/estatistica/populacao/trabalhoerendimento/pnad2008/brasilpnad2008.pdf

[10] Brasil Instituto Brasileiro de Geografia e Estatística (2010) Dados preliminares do Censo 2010 já revelam mudanças na pirâmideetária brasileira. IBGE, Rio de Janeiro. http://saladeimprensa.ibge.gov.br/noticias?view=noticia\&id=1\&idnoticia=1722\&busca=1\&t=dados-preliminares-cens o-2010-ja-revelam-mudancas-piramide-etária-brasileira

[11] Brasil. Instituto Brasileiro de Geografia e Estatística. POF 2008-2009: Desnutrição cai e peso das crianças brasileiras ultrapassa padrão internacional.

http://www.ibge.gov.br/home/presidencia/noticias/noticia visualiza.php?id noticia=1699\&amp;id pagina=1

[12] Brasil. Instituto Brasileiro de Geografia e Estatística (2010) Censo demográfico 2010: Resultados gerais da amostra. IBGE, Rio de Janeiro. http://biblioteca.ibge.gov.br/visualizacao/periodicos/99/cd_2010_resultados_gerais_amostra.pdf

[13] Losina, E., Walensky, R.P., Reichmann, W.M., Holt, H.L., Gerlovin, H., Solomon, D.H., et al. (2011) Impact of Obesity and Knee Osteoarthritis on Morbidity and Mortalityin Older Americans. Annals of Internal Medicine, 154, 217-226. http://dx.doi.org/10.7326/0003-4819-154-4-201102150-00001

[14] Zhang, W. (2010) Risk Factors of Knee Osteoarthritis-Excellent Evidence but Little Has Been Done. Osteoarthritis and Cartilage, 18, 1-2. http://dx.doi.org/10.1016/j.joca.2009.07.013

[15] Zhang, W., Moskowitz, R.W., Nuki, G., Abramson, S., Altman, R.D., Arden, N., et al. (2008) OARSI Recommendations for the Management of Hip and Knee Osteoarthritis, Part II: OARSI Evidence-Based, Expert Consensus Guidelines. Osteoarthritis and Cartilage, 16, 137-162. http://dx.doi.org/10.1016/j.joca.2007.12.013

[16] Bliddal, H., Leeds, A.R., Stigsgaard, L., Astrup, A. and Christensen, R. (2011) Weight Loss as Treatment for Knee Osteoarthritis Symptoms in Obese Patients: 1 Year Results from a Randomized Controlled Trial. Annals of the Rheumatic Diseases, 70, 1798-1803. http://dx.doi.org/10.1136/ard.2010.142018

[17] Zhang, W., Nuki, G., Moskowitz, R.W., Abramson, S., Altman, R.D., et al. (2010) OARSI Recommendations for the Management of Hip and Knee Osteoarthritis Part III: Changes in Evidence Following Systematic Cumulative Update of Research Published through January 2009. Osteoarthritis and Cartilage, 18, 476-499. http://dx.doi.org/10.1016/j.joca.2010.01.013

[18] Bruyère, O., Cooper, C., Pelletier, J.P., Branco, J., Luisa Brandi, M., Guillemin, F., et al. (2014) An Algorithm Recommendation for the Management of Knee Osteoarthritis in Europe and Internationally: A Report from a Task Force of the European Society for Clinical and Economic Aspects of Osteoporosis and Osteoarthritis (ESCEO). Seminars in Arthritis and Rheumatism, 44, 253-263. http://dx.doi.org/10.1016/j.semarthrit.2014.05.014

[19] Shin, D. (2014) Association between Metabolic Syndrome, Radiographic Knee Osteoarthritis, and Intensity of Knee Pain: Results of a National Survey. The Journal of Clinical Endocrinology \& Metabolism, 99, 3177-3183. http://dx.doi.org/10.1210/jc.2014-1043

[20] Schrieber, L. and Colley, M. (2004) Patient Education. Best Practice \& Research Clinical Rheumatology, 18, $465-476$. http://dx.doi.org/10.1016/j.berh.2004.03.008

[21] Buszewicz, M., Rait, G., Griffin, M., Nazareth, I., Patel, A., Atkinson, A., et al. (2006) Self Management of Arthritis in Primary Care: Randomised Controlled Trial. BMJ, 333, 879. http://dx.doi.org/10.1136/bmj.38965.375718.80

[22] Rosemann, T., Joos, S., Laux, G., Gensichen, J. and Szecsenyi, J. (2007) Case Management of Arthritis Patients in Primary Care: A Cluster-Randomized Controlled Trial. Arthritis \& Rheumatology, 57, 1390-1397. http://dx.doi.org/10.1002/art.23080

[23] Ravaud, P., Flipo, R.M., Boutron, I., Roy, C., Mahmoudi, A., et al. (2009) ARTIST (Osteoarthritis Intervention Standardized) Study of Standardized Consultation versus Usual Care for Patients with Osteoarthritis of the Knee in Primary care in France: Pragmatic Randomised Controlled Trial. BMJ, 338, b421. http://dx.doi.org/10.1136/bmj.b421

[24] Plapler, P.G., Saron, T.R.P. and Rezende, M.U. (2014) Education and Physical Activity in Osteoporosis. Journal of Osteoporosis and Physical Activity, 2, 118.

[25] Moher, D., Hopewell, S., Schulz, K.F., Montori, V., Gotzsche, P.C., Devereaux, P.J., et al. (2012) CONSORT 2010 Explanation and Elaboration: Updated Guidelines for Reporting Parallel Group Randomised Trials. International Journal of Surgery, 10, 28-55. http://dx.doi.org/10.1016/j.ijsu.2011.10.001 
[26] Altman, R., Asch, E., Bloch, D., Bole, G., Borenstein, D., Brandt, K., et al. (1986) Development of Criteria for the Classification and Reporting of Osteoarthritis: Classification of Osteoarthritis of the Knee. Arthritis \& Rheumatology, 29, 1039-1049. http://dx.doi.org/10.1002/art.1780290816

[27] Bellamy, N., Buchanan, W.W., Goldsmith, C.H., Campbell, J. and Stitt, L.W. (1988) Validation Study of WOMAC: A Health Status Instrument for Measuring Clinically Important Patient Relevant Outcomes to Antirheumatic Therapy in Patient with Osteoarthritis of the Hip or Knee. The Journal of Rheumatology, 15, 1833-1840.

[28] Lequesne, M.G. (1997) The Algofunctional Indices for Hip and Knee Osteoarthritis. The Journal of Rheumatology, 24, 779-781.

[29] McHorney, C.A., Ware, J.E. and Raczek, A.E. (1993) The MOS 36-Item Short-Form Health Survey (SF-36): II. Psychometric and Clinical Tests of Validity in Measuring Physical and Mental Health Construct. Medical Care, 31, 247-263. http://dx.doi.org/10.1097/00005650-199303000-00006

[30] Kellgren, J.H. and Lawrence, J.S. (1957) Radiological Assessment of Rheumatoid Arthritis. Annals of the Rheumatic Diseases, 16, 485-493. http://dx.doi.org/10.1136/ard.16.4.485

[31] Rezende, M.U., Campos, G.C., Pailo, A.F., Frucchi, R., Pasqualin, T. and Camargo, O.P. (2013) PARQVE-Project Arthritis Recovering Quality of Life by Means of Education Short-Term Outcome in a Randomized Clinical Trial. Journal of Arthritis, 2, 133.

[32] Sanghi, D., Srivastava, R.N., Singh, A., Kumari, R., Mishra, R. and Mishra, A. (2011) The Association of Anthropometric Measures and Osteoarthritis Knee in Non-Obese Subjects: A Cross Sectional Study. Clinics, 66, $275-279$. http://dx.doi.org/10.1590/S1807-59322011000200016

[33] Christensen, R., Bartels, E.M., Astrup, A. and Bliddal, H. (2007) Effect of Weight Reduction in Obese Patients Diagnosed with Knee Osteoarthritis: A Systematic Review and Meta-Analysis. Annals of the Rheumatic Diseases, 66, 433-439. http://dx.doi.org/10.1136/ard.2006.065904 